\title{
Mathematical analysis of single queue multi server and multi queue multi server queuing models: comparison study
}

\author{
S. Vijay Prasad ${ }^{1 *}$, V.H. Badshah ${ }^{2}$, Tariq Ahmad Koka ${ }^{1}$ \\ ${ }^{1}$ Research scholar, School of Studies in Mathematics, Vikram University, Ujjain, M. P, 456010, India \\ ${ }^{2}$ Professor \& Head, School of Studies in Mathematics, Vikram University, Ujjain, M. P, 456010, India \\ *Corresponding author E-mail: indorevijay@gmail.com
}

Copyright $(92015$ S. Vijay Prasad et al. This is an open access article distributed under the Creative Commons Attribution License, which permits unrestricted use, distribution, and reproduction in any medium, provided the original work is properly cited.

\begin{abstract}
In the research paper entitled Mathematical Analysis of Single Queue Multi Server and Multi Queue Multi Server Queuing Model, Prasad and Badshah [7] were proved that single queue multi server model is better than multi queue multi server model, and discussed the relation between the performance measures of these two models, and derive the mathematical equations. In this paper we derive the total cost with assumption of certain Waiting cost in both cases. Also, prove that the expected total cost is less for single queue multi server model as comparing with multi queue multi server model.
\end{abstract}

Keywords: M/M/1 Model; M/M/S Model; Single Queue - Multi Server and Multi Queue - Multi Server Queuing Models; Service Cost; Waiting Cost; Expected Total Cost.

\section{Introduction}

Queuing theory is the study of queues or waiting lines. Some of the analysis can be derived using queuing theory include expected waiting time in the queue, the average time in the system, the expected queue length, the expected number of customers served at one time, the probability of balking customers, as well as the probability of the system to be in certain states, such as empty or full. Waiting lines are a common sight in super markets. Hence, queuing theory is suitable to be applied in a super market setting since it has an associated queue or waiting line, where customers who cannot be served immediately have to queue (wait) for service [1].

Along with the continuous development of market economy, more supermarkets appear in cities and towns [8]. Super markets have done great efforts to increase the marketing, but most of them are facing a serious problem which is customer queuing, which led to low service rate of checkout stands, poor business environment and number of high quality customers and potential customers are lost and so on [3]. Checkout stands are the service windows of supermarkets, which are not only reflecting supermarkets images but also, associate with supermarkets service quality and business efficiency. The long queue waiting for checkout is not ideal purchasing environment for people. Most of people prefer to give up or going away instead of waiting in queue, with similar quality and price, service quality is the key for winning the competition. However, few checkout stands may lead to serious waiting affecting service and causing loses of customers. The most direct complained from customers do not satisfied, with checkout stands is that the customers wait for a long time and do not get good service [9]. The problem of customers waiting for the shortest time is studied by means of queuing models, the measure to reduce the time of the customer's queues is obtained to achieve the goal of the people oriented and the greatest effectiveness of the checkout stands of supermarkets. Generally we see multi queue - multi server model in the super markets, Xiao and Zhang [3] were proved that a line better than more lines. Prasad and Badshah [7] were proved that single queue - multi server model is better than multi queue multi server model and discussed the mathematical relations of the performance measures of both queuing models. The expected number for customers waiting in the queue $\left(\mathrm{L}_{\mathrm{q}}\right)$ is less in the case of single queue as compare with case of $S$ queues. The expected waiting time of the customer in the queue $\left(\mathrm{W}_{\mathrm{q}}\right)$ is less in the case of single queue as compare with 
case of $S$ queues. The expected waiting time of the customer in the system $\left(\mathrm{W}_{\mathrm{s}}\right)$ is less in the case of single queue as compare with case of $\mathrm{S}$ queues. The expected number of customers waiting in the system $\left(\mathrm{L}_{\mathrm{q}}\right)$ is greater in the case of single queue as compare with case of $\mathrm{S}$ queues. In this paper we derive the total cost with assumption of certain Waiting cost in both cases and prove that the expected total cost is less for single queue multi server model as comparing with multi queue multi server model.

\section{Materials and methods}

\subsection{The basic indexes of the queuing systems}

$\mathrm{n}=$ Number of customers in the system

$\lambda=$ Mean arrival rate

$\mu=$ Mean service rate per busy server

$\rho=$ Expected fraction of time for which server is busy

$\mathrm{P}_{\mathrm{n}}=$ Steady state probability of exactly $\mathrm{n}$ customers in the system

$\mathrm{L}_{\mathrm{q}}=$ Expected number of customers waiting in the queue (i.e. queue length)

$\mathrm{L}_{\mathrm{s}}=$ Expected number of customers in the system (waiting + being served)

$\mathrm{w}_{\mathrm{q}}=$ Expected waiting time for a customer in the queue

$\mathrm{W}_{\mathrm{s}}=$ Expected waiting time for a customer in the system (waiting + being served)

\subsection{Queuing models}
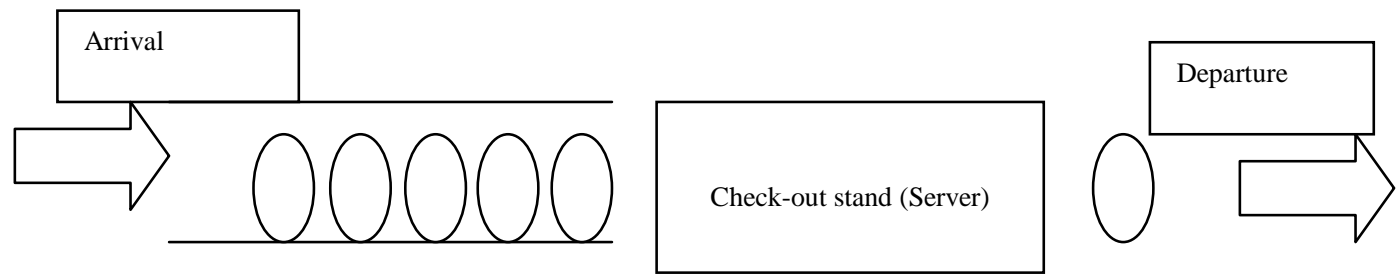

Fig. 1: Single Queue - Single Server Model.
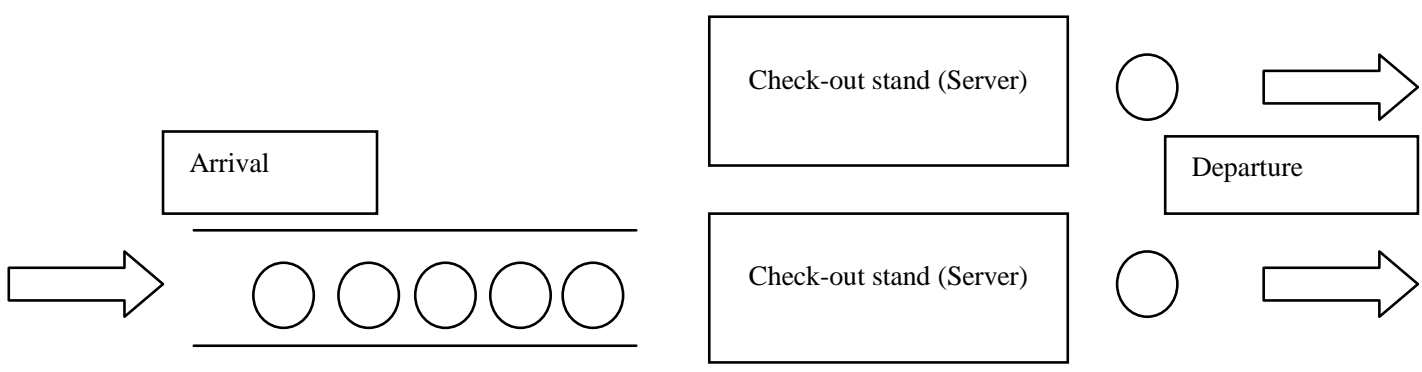

Check-out stand (Server)

Fig. 2: Single Queue - Multi Server Model
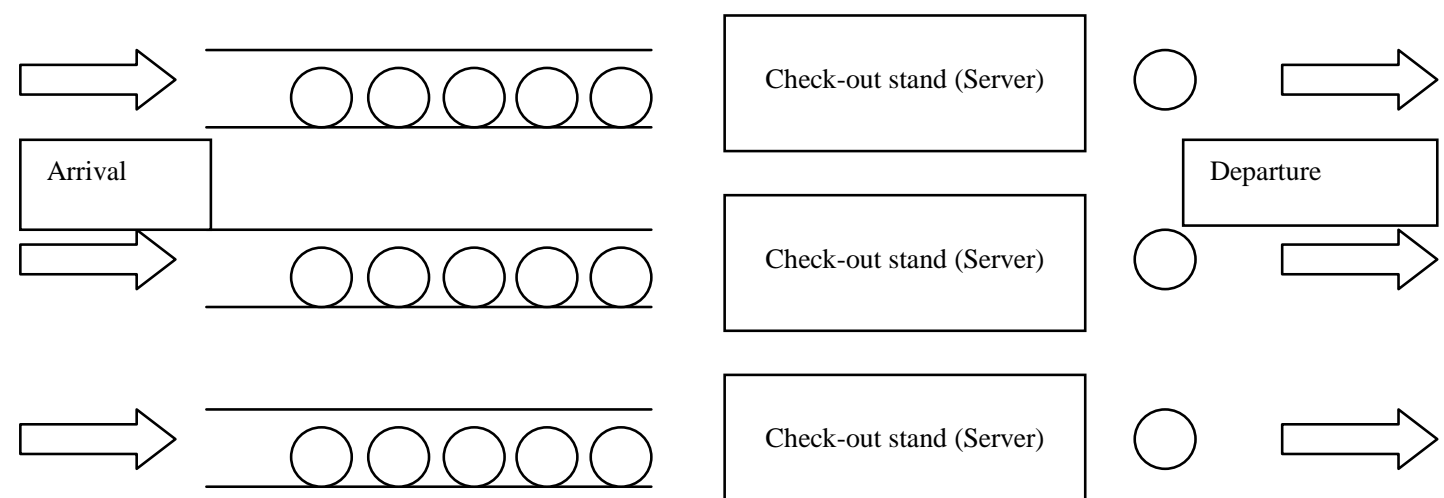

Check-out stand (Server)

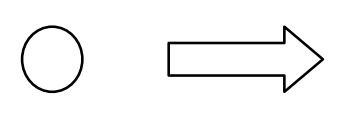

Fig. 3: Multi Queue - Multi Server 


\section{3. $M / M / 1$ Model (single server queuing model)}

In this model Arrivals are described by Poisson probability distribution, and come from and infinite population, queue discipline is first come first serve, single server and service time follows exponential distribution. This queuing system can be applied to a wide variety of problems as any system with a large number of independent customers, and can be approximated as a Poisson process: $\mathrm{P}_{\mathrm{n}}(\mathrm{n}=0,1,2 .$.$) is the probability distribution of the queue length.$

Expected number of customers in the system (waiting + being served)

$\mathrm{L}_{\mathrm{s}}=\sum_{\mathrm{n}=0}^{\infty} \mathrm{nP}_{\mathrm{n}}=\sum_{\mathrm{n}=0}^{\infty} \mathrm{n}(1-\rho) \rho^{\mathrm{n}}$,

$=\frac{\rho}{(1-\rho)}=\frac{\lambda}{\mu-\lambda}$

\subsection{M/M/S Model (multi server queuing system)}

For this queuing system, it is assumed that arrivals follow a Poisson probability distribution at an average rate of $\lambda$ customers per unit of time and are served on a first come first served basis by any of the servers. The service times are distributed exponentially with an average of $\mu$ customers per unit of time. It is further assumed that only one queue is formed. If there are $\mathrm{n}$ customers in the queuing system at any point in time, then following two cases may arise:

i) If $\mathrm{n}<s$ (number of customers in the system is less than the number of servers), then there will be no queue. However, $(s-n)$ numbers of servers are not busy.

The combined service rate will then be: $\mu_{\mathrm{n}}=\mathrm{n} \mu ; \mathrm{n}<s$

ii) If $\mathrm{n} \geq \mathrm{s}$, (number of customers in the system is more than or equal to the number of servers) then all servers will be busy and the maximum number of customers in the queue will be

$(n-s)$. The combined service rate will be

$\mu_{\mathrm{n}}=\mathrm{s} \mu ; \mathrm{n} \geq \mathrm{s}$

Thus to derive the result for this model, we have

$\lambda_{\mathrm{n}}=\lambda$ for all $\mathrm{n} \geq 0 \quad \mu_{\mathrm{n}}=\left\{\begin{array}{c}\mathrm{n} \mu ; \mathrm{n}<\mathrm{s} \\ \mu_{\mathrm{n}}=\mathrm{s} \mu ; \mathrm{n} \geq \mathrm{s}\end{array}\right.$

The probability $\mathrm{P}_{\mathrm{n}}$ of $\mathrm{n}$ customers in the queuing system is given by

$P_{n}=\left\{\begin{array}{l}\frac{\rho^{n}}{n !} P_{0} ; n \leq s \\ \frac{\rho^{n}}{s ! s^{n-s}} P_{0} ; n>s\end{array}\right.$

$P_{0}=\left[\sum_{n=0}^{s-1} \frac{1}{n !}(\lambda / \mu)^{n}+\frac{1}{s !}(\lambda / \mu)^{s}\left(\frac{s \mu}{s \mu-\lambda}\right)\right]^{-1}$

Expected number of customers waiting in the queue (i.e. queue length)

$L_{q}=\left[\frac{1}{(s-1) !}\left(\frac{\lambda}{\mu}\right)^{s} \frac{\lambda \mu}{(s \mu-\lambda)^{2}}\right] P_{0}$

Expected number of customers in the system

$\mathrm{L}_{s}=L_{q}+\frac{\lambda}{\mu}$

\subsection{Expected total cost for $M / M / 1$ and $M / M / S$ model}

Service level is the function of two conflicting costs:

i) Cost of offering the service to the customers

ii) Cost of delay in offering service to the customers

Economic analysis of these costs helps the management to make a trade-off between the increased costs of providing better service and the decreased waiting time costs of customers derived from providing that service.

Expected service cost $\mathrm{E}(\mathrm{SC})=\mathrm{sC}_{\mathrm{s}}$

Where $s$ is number of servers and $C_{s}$ is service cost of each server 
Expected waiting cost in the system $\mathrm{E}(\mathrm{WC})=\mathrm{L}_{\mathrm{s}} \mathrm{C}_{\mathrm{w}}$

Where $\mathrm{L}_{\mathrm{s}}=$ Expected number of customers in the system and

$C_{w}$ Is cost of waiting by the customer

Expected total cost in the case single queue - single server model (i.e. $M / M / 1$ model)

Expected total cost $\mathrm{E}(\mathrm{TC})=\mathrm{E}(\mathrm{SC})+\mathrm{E}(\mathrm{WC})=\mathrm{C}_{\mathrm{s}}+\mathrm{L}_{\mathrm{s}} \mathrm{C}_{\mathrm{w}}$

Expected total cost in the case multi queue - multi server model (i.e. $S$ individual $M / M / 1$ models)

$\mathrm{E}(\mathrm{TC})=\mathrm{S}(\mathrm{E}(\mathrm{SC})+\mathrm{E}(\mathrm{WC}))=\mathrm{S}\left(\mathrm{C}_{\mathrm{s}}+\mathrm{L}_{\mathrm{s}} \mathrm{C}_{\mathrm{w}}\right)$

Expected total cost in the case single queue - multi server model (i.e. $M / M / S$ model)

Expected total cost $\mathrm{E}(\mathrm{TC})=\mathrm{E}(\mathrm{SC})+\mathrm{E}(\mathrm{WC})=\mathrm{sC}_{\mathrm{s}}+\mathrm{L}_{\mathrm{s}} \mathrm{C}_{\mathrm{w}}$

\section{Results and discussions}

If we consider $S$ number of checkout stands in the supermarket, and the customer's arrival rate is $\lambda$ and the service rate of each checkout stand is $\mu$, if we try to find the values of $L_{q}, L_{s}, W_{q}$ and $W_{s}$ in two cases and comparing all these four characteristics in each case.

- If there is only one queue then the system considered as $M / M / S$ queuing system, in this case we will use multi server queuing model to find $L_{q}, L_{s}, W_{q}$ and $W_{s}$.

- If there are $\mathrm{S}$ queues in the system the queuing system considered as $\mathrm{S}$ isolated $M / M / 1$ queuing systems, in this case we will use single server queuing model to find $L_{q}, L_{s}, W_{q}$ and $W_{s}$, in this case the customer's arrival rate become $\frac{\lambda}{S}$.

In the research paper entitled Mathematical Analysis of Single Queue Multi Server and Multi Queue Multi Server Queuing Model Prasad and Badshah [7] proved that single queue multi server model is better than multi queue multi server model and we discuss the relation between the performance measures of these two models and derive the mathematical equations are as follows:

i) The expected number of customers waiting in the queue $\left(L_{q}\right)$ is less in the case of single queue as we compare with case of $S$ queues that is

$L_{q}$ (In the case of one queue $)<L_{q}$ (In the case of S Queues)

$\frac{\rho^{S+1}}{(S-\rho)\left[(S-1) !(S-\rho) \sum_{n=0}^{S-1} \frac{\rho^{n}}{n !}+\rho^{S}\right]}<\frac{\rho^{2}}{S(S-\rho)}$

ii) The expected waiting time of the customer in the queue $\left(\mathrm{W}_{\mathrm{q}}\right)$ is less in the case of single queue as we compare with case of $S$ queues that is

$W_{q}$ (In the case of one queue) $<W_{q}$ (In the case of S Queues)

$\frac{\rho^{S+1}}{\lambda(S-\rho)\left[(S-1) !(S-\rho) \sum_{n=0}^{S-1} \frac{\rho^{n}}{n !}+\rho^{S}\right]}<\frac{\rho^{2}}{\lambda S(S-\rho)}$

iii) The expected waiting time of the customer in the system $\left(\mathrm{W}_{\mathrm{s}}\right)$ is less in the case of single queue as we compare with case of $S$ queues that is

$W_{s}$ (In the case of one queue) $<W_{s}$ (In the case of S Queues)

$\frac{\rho^{S+1}}{\lambda(S-\rho)\left[(S-1) !(S-\rho) \sum_{n=0}^{S-1} \frac{\rho^{n}}{n !}+\rho^{S}\right]}+\frac{1}{\mu}<\frac{\rho^{2}}{\lambda S(S-\rho)}+\frac{1}{\mu}$

iv) The expected number of customers waiting in the system $\left(\mathrm{L}_{\mathrm{q}}\right)$ is greater in the case of single queue as we compare with case of $S$ queues that is

$L_{S}$ (In the case of one queue) $>L_{S}$ (In the case of S Queues)

$\frac{\rho^{S+1}}{(S-\rho)\left[(S-1) !(S-\rho) \sum_{n=0}^{S-1} \frac{\rho^{n}}{n !}+\rho^{S}\right]}+\rho>\frac{\rho}{(S-\rho)}$ 
In this paper we derive the total cost with assumption of certain Waiting cost in both cases and prove that the expected total cost is less for single queue multi server model as comparing with multi queue multi server model.

\subsection{Case -I.}

If we consider $s=2$ checkout stands in the supermarket, the customer's arrival rate is $\lambda=60$ and the service rate of each checkout stand is $\mu=40$ to find the expected total cost assuming that service cost is Rs. 200 and waiting cost is Rs. 250 in each case

- If there is only one queue then the system considered as $M / M / 2$ queuing system in this case we will use multi server queuing model to find expected total cost.

- If there are two queues in the system the queuing system considered as two isolated $M / M / 1$ queuing systems, in this case we will use single server queuing model to find expected total cost and the customer's arrival rate become $\frac{\lambda}{2}=30$.

For the case of only one queue in the system

$\lambda=60, \mu=40, s=2$

$P_{0}=\left[\sum_{n=0}^{s-1} \frac{1}{n !}(\lambda / \mu)^{n}+\frac{1}{s !}(\lambda / \mu)^{s}\left(\frac{s \mu}{s \mu-\lambda}\right)\right]^{-1}$

$P_{0}=\left[\sum_{n=0}^{2-1} \frac{1}{n !}(60 / 40)^{n}+\frac{1}{2 !}(60 / 40)^{2}\left(\frac{80}{80-60}\right)\right]^{-1}, P_{0}=[4.75]^{-1}, P_{0}=0.14286$

$L_{q}=\left[\frac{1}{(s-1) !}\left(\frac{\lambda}{\mu}\right)^{S} \frac{\lambda \mu}{(s \mu-\lambda)^{2}}\right] P_{0}$

$=\left[\frac{1}{(2-1) !}\left(\frac{60}{40}\right)^{2} \frac{2400}{(80-60)^{2}}\right](0.21)=1.9286$

$L_{s}=L_{q}+\frac{\lambda}{\mu}=1.9286+\frac{60}{40}=3.4286$

For the case of two queues in the system

$\lambda=\frac{60}{2}=30, \mu=40$,

$L_{s}=\frac{\lambda}{\mu-\lambda}=\frac{30}{40-30}=3$

The expected total cost in the case of one queue (single queue - multi server)

Expected total cost $\mathrm{E}(\mathrm{TC})=\mathrm{E}(\mathrm{SC})+\mathrm{E}(\mathrm{WC})=\mathrm{sC}_{\mathrm{s}}+\mathrm{L}_{\mathrm{s}} \mathrm{C}_{\mathrm{w}}$

$=2 * 200+3.4286 * 250=1257.15$

The expected total cost in the case of two queues (multi queue - multi server)

Expected total cost $\mathrm{E}(\mathrm{TC})=\mathrm{S}(\mathrm{E}(\mathrm{SC})+\mathrm{E}(\mathrm{WC}))=\mathrm{S}\left(\mathrm{C}_{\mathrm{s}}+\mathrm{L}_{\mathrm{s}} \mathrm{C}_{\mathrm{w}}\right)$

$=2(200+3 * 250)=1900$

From (13) and (14) we can conclude that

The expected total cost in the case of one queue (single queue - multi server) less than the expected total cost in the case of two queues (multi queue - multi server).

\subsection{Case-II.}

Again we consider $s=3$ checkout stands in the supermarket, the customer's arrival rate is $\lambda=60$ and the service rate of each checkout stand is $\mu=40$ to find the expected total cost assuming that service cost is Rs. 200 and waiting cost is Rs. 250 in each. 
- If there is only one queue then the system will be considered as $M / M / 3$ queuing system, in this case we will use multi server queuing model to find expected total cost.

- If there are three queues in the system the queuing system considered as three isolated $M / M / 1$ queuing systems, in this case we will use single server queuing model to find expected total cost in this case the customer's arrival rate become $\frac{\lambda}{3}=20$.

For the case of only one queue in the system

$\lambda=60, \mu=40, s=3$

$P_{0}=0.21$

$L_{S}=1.736$

For the case of three queues in the system

$\lambda=\frac{60}{3}=20, \mu=40$,

$L_{s}=1$

The expected total cost in the case of one queue (single queue - multi server).

Expected total cost $\mathrm{E}(\mathrm{TC})=\mathrm{E}(\mathrm{SC})+\mathrm{E}(\mathrm{WC})=\mathrm{sC}_{\mathrm{s}}+\mathrm{L}_{\mathrm{s}} \mathrm{C}_{\mathrm{w}}$

$=3 * 200+1.736 * 250=1034$

The expected total cost in the case of two queues (multi queue - multi server).

Expected total cost $\mathrm{E}(\mathrm{TC})=\mathrm{S}(\mathrm{E}(\mathrm{SC})+\mathrm{E}(\mathrm{WC}))=\mathrm{s}\left(\mathrm{C}_{\mathrm{s}}+\mathrm{L}_{\mathrm{s}} \mathrm{C}_{\mathrm{w}}\right)$

$=3(200+1 * 250)=1350$

From (15) and (16) we can conclude that

The expected total cost in the case of one queue (single queue - multi server) less than the expected total cost in the case of two queues (multi queue - multi server).

\subsection{Case -III}

As we did this concept is further lead us as follows, if we consider $S$ number of checkout stands in the supermarket and the customer's arrival rate is $\lambda$ and the service rate of each checkout stand is $\mu$, if we try to find expected total cost in two cases and comparing the expected total cost in each case.

- If there is only one queue then the system considered as $M / M / S$ queuing system, in this case we will use multi server queuing model to expected total cost.

- If there are $\mathrm{S}$ queues in the system the queuing system considered as $\mathrm{S}$ isolated $M / M / 1$ queuing systems, in this case we will use single server queuing model to find expected total cost, in this case the customer's arrival rate become $\frac{\lambda}{S}$.

For the case of only one queue in the system

$$
\begin{aligned}
P_{0} & =\left[\sum_{n=0}^{s-1} \frac{1}{n !}(\lambda / \mu)^{n}+\frac{1}{s !}(\lambda / \mu)^{s}\left(\frac{s \mu}{s \mu-\lambda}\right)\right]^{-1} \\
L_{q} & =\left[\frac{1}{(s-1) !}\left(\frac{\lambda}{\mu}\right)^{s} \frac{\lambda \mu}{(s \mu-\lambda)^{2}}\right] P_{0} \\
L_{s} & =L_{q}+\frac{\lambda}{\mu}
\end{aligned}
$$

For the case of two queues in the system

$$
\lambda=\frac{\lambda}{s}, \mu=\mu,
$$


$L_{s}=\frac{\lambda}{s \mu-\lambda}$

The expected total cost in the case of one queue (single queue - multi server) less than the expected total cost in the case of two queues (multi queue - multi server) that is

$E(T C)$ (In the case of one queue) $<E(T C)$ (In the case of S Queues).

$\mathrm{sC}_{\mathrm{s}}+\mathrm{L}_{\mathrm{s}} \mathrm{C}_{\mathrm{w}}<\mathrm{s}\left(\mathrm{C}_{\mathrm{s}}+\mathrm{L}_{\mathrm{s}} \mathrm{C}_{\mathrm{w}}\right)$

$\mathrm{L}_{\mathrm{s}}<\mathrm{sL}_{\mathrm{s}}$

$\frac{\rho^{S+1}}{(S-\rho)\left[(S-1) !(S-\rho) \sum_{n=0}^{S-1} \frac{\rho^{n}}{n !}+\rho^{S}\right]}+\rho<\frac{s \rho}{(S-\rho)}$

$\frac{\rho\left[\rho^{S}+(S-\rho)\left[(S-1) !(S-\rho) \sum_{n=0}^{S-1} \rho^{n}+\rho^{s}\right]\right]}{(S-\rho)\left[(S-1) !(S-\rho) \sum_{n=0}^{S-1} \frac{\rho^{n}}{n !}+\rho^{S}\right]}<\frac{s \rho}{(S-\rho)}$

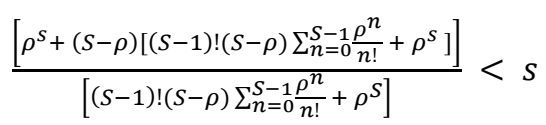

$-\rho^{s+1}+(s-\rho)^{2}(s-1) ! \sum_{n=0}^{S-1} \frac{\rho^{n}}{n !}-s(s-1) !(s-\rho) \sum_{n=0}^{S-1} \frac{\rho^{n}}{n !}<0$

$-\rho^{s+1}+\rho^{s}-\rho(s-1) !(s-\rho) \sum_{n=0}^{S-1} \frac{\rho^{n}}{n !}<0$

$-\rho^{s}+\rho^{s-1}-(s-1) !(s-\rho) \sum_{n=0}^{S-1} \frac{\rho^{n}}{n !}<0$

Let $f(s)=-\rho^{s}+\rho^{s-1}-(s-1) !(s-\rho) \sum_{n=0}^{S-1} \frac{\rho^{n}}{n !}<0$, forall $s>1$

The equation can be proved by using Mathematical Induction formula

If $S=2$

$f(2)=-\rho^{2}+\rho^{2-1}-(2-1) !(2-\rho) \sum_{n=0}^{2-1} \frac{\rho^{n}}{n !}$

$f(2)=-\rho^{2}+\rho-(2-\rho)(1+\rho)$

$f(2)=-\rho^{2}+\rho-2-\rho+\rho^{2}$

$f(2)=-2<0$

Now we assume that which is true for $S=k$

$f(k)=-\rho^{k}+\rho^{k-1}-(k-1) !(k-\rho) \sum_{n=0}^{k-1} \frac{\rho^{n}}{n !}<0$

Now we have to prove that which is true for $S=k+1$

$$
\begin{aligned}
& f(k+1)=-\rho^{k+1}+\rho^{k+1-1}-(k+1-1) !(k+1-\rho) \sum_{n=0}^{k+1-1} \frac{\rho^{n}}{n !} \\
& f(k+1)=-\rho^{k+1}+\rho^{k}-(k) !(k+1-\rho) \sum_{n=0}^{k} \frac{\rho^{n}}{n !} \\
& f(k+1)=-\rho^{k+1}+\rho^{k}-(k) !(k+1-\rho)\left[\sum_{n=0}^{k-1} \frac{\rho^{n}}{n !}+\frac{\rho^{k}}{k !}\right]
\end{aligned}
$$


$f(k+1)=-\rho^{k+1}+\rho^{k}-(k+1-\rho)\left[(k) ! \sum_{n=0}^{k-1} \frac{\rho^{n}}{n !}+(k) ! \frac{\rho^{k}}{k !}\right]$

$f(k+1)=-\rho^{k+1}+\rho^{k}-(k+1-\rho)(k) ! \sum_{n=0}^{k-1} \frac{\rho^{\mathrm{n}}}{\mathrm{n} !}-(\mathrm{k}+1-\rho) \rho^{\mathrm{k}}$

$\mathrm{f}(\mathrm{k}+1)=-\left[(\mathrm{k}+1-\rho)(\mathrm{k}) ! \sum_{\mathrm{n}=0}^{\mathrm{k}-1} \frac{\rho^{\mathrm{n}}}{\mathrm{n} !}+\mathrm{k} \rho^{\mathrm{k}}\right]<0$

Hence it is true for all $\mathrm{S}>1$

\section{Conclusion}

In this paper we derived the total cost with assumption of certain Waiting cost in both cases, and proved that the expected total cost is less for single queue multi server model as comparing with multi queue multi server model. Mathematically it has been proved by the examples.

\section{References}

[1] Dharmawirya. M, Adi. E, Case Study for Restaurant Queuing Model, International Conference on Management and Artificial Intelligence, IPEDR vol.6, 2011, pp. 52-55

[2] Hamdy A.Taha, Operations research: an introduction, 8th edition, Pearson Education, Inc., 2007, pp. 557-558

[3] Huimin Xiao, Guozheng Zhang, The queuing application in bank service optimization, IEEE, 2010, pp. 1097- 1100

[4] Prem Kumar Gupta, D.S. Hira, Operations research, revised edition, S. Chand, 2008, pp. 903-910

[5] Song hongna lect., Simulation of banks queuing system based on witness, International Conference on Computer Application and System Modeling, Vol.15, 2010, pp. 402-405

[6] Vijay Prasad. S, Badshah. V.H, Pradeep Porwal, Decision Making by M/M/S Queuing Model: A Case Study-I, International Journal of Pure and Applied Mathematical Sciences, Volume 7, 2014, pp. 137-143

[7] Vijay Prasad. S, Badshah. V.H, Mathematical Analysis of Single Queue Multi Server and Multi Queue Multi Server Queuing Model, IJAEET, Vol. 5, No. 6, 2014, pp. $264-273$

[8] X.X. Zaho, Queuing theory with bank management innovation, Modern finance, No.3, 2007, pp. 9- 10

[9] Yuejian Jie, The optimal supermarket service, International Journal of Business Management, Vol.5, No.2, 2010, pp. 128-129 http://dx.doi.org/10.5539/ijbm.v5n2p128. 